\title{
Stability of ground-states for a system of $M$ coupled semilinear Schrödinger equations
}

\author{
Simão Correia
}

\begin{abstract}
We focus on the study of the stability properties of groundstates for the system of $M$ coupled semilinear Schrödinger equations with power-type nonlinearities and couplings. Our results are generalizations of the theory for the single equation and the technique used is a simplification of the original one. Depending on the power of the nonlinearity, we may observe stability, instability and weak instability. We also obtain results for three distinct classes of bound-states, which is a special feature of the $M \geqslant 2$ case.
\end{abstract}

Mathematics Subject Classification. 35Q55, 35B35, 35B40.

Keywords. Coupled semilinear Schrödinger equations, Ground-states, Stability.

\section{Introduction}

In this work, we consider the system of $M$ coupled semilinear Schrödinger equations

$$
i\left(v_{i}\right)_{t}+\Delta v_{i}+\sum_{j=1}^{M} k_{i j}\left|v_{j}\right|^{p+1}\left|v_{i}\right|^{p-1} v_{i}=0, \quad i=1, \ldots, M
$$

where $V=\left(v_{1}, \ldots, v_{M}\right): \mathbb{R}^{+} \times \mathbb{R}^{N} \rightarrow \mathbb{R}^{M}, k_{i j} \in \mathbb{R}, k_{i j}=k_{j i}$, and $0<p<$ $4 /(N-2)^{+}$(we use the convention $4 /(N-2)^{+}=+\infty$, if $N=1,2$, and $4 /(N-2)^{+}=4 /(N-2)$, if $\left.N \geqslant 3\right)$. Given $1 \leqslant i \neq j \leqslant M$, if $k_{i j} \geqslant 0$, one says that the coupling between the components $v_{i}$ and $v_{j}$ is attractive; if $k_{i j}<0$, it is repulsive. The Cauchy problem for $V_{0} \in\left(H^{1}\left(\mathbb{R}^{N}\right)\right)^{M}$ is locally well-posed and, letting $T_{\max }\left(V_{0}\right)$ be the maximal time of existence of the solution with initial data $V_{0}$ : if $T_{\max }\left(V_{0}\right)<\infty$, then $\lim _{t \rightarrow T_{\max }\left(V_{0}\right)}\|\nabla V(t)\|_{2}=+\infty$.

In the case $M=1$ and $k=1$, we obtain the nonlinear Schrödinger equation

$$
i v_{t}+\Delta v+|v|^{2 p} v=0
$$


When we look for nontrivial periodic solutions of the form $V=e^{i t} U$, with $U=\left(u_{1}, \ldots, u_{M}\right) \in\left(H^{1}\left(\mathbb{R}^{N}\right)\right)^{M}$ (called bound-states), we are led to the study of the system

$$
\Delta u_{i}-u_{i}+\sum_{j=1}^{M} k_{i j}\left|u_{j}\right|^{p+1}\left|u_{i}\right|^{p-1} u_{i}=0 \quad i=1, \ldots, M .
$$

Especially relevant, for both physical and mathematical reasons, are the bound-states which have minimal action among all bound-states, the so-called ground-states. In the scalar case, one may prove that there is a unique groundstate (modulo translations and rotations).

In a recent paper $([2])$, we proved the existence of ground-states of (MNLS) under the assumption

$$
\left\{U \in\left(H^{1}\left(\mathbb{R}^{N}\right)\right)^{M}: \sum_{i, j=1}^{M} k_{i j}\left\|u_{i} u_{j}\right\|_{p+1}^{p+1}>0\right\} \neq \emptyset .
$$

Note that this assumption is a necessary condition for existence of boundstates, since, multiplying (1.1) by $U$ and integrating over $\mathbb{R}^{N}$, one obtains

$$
\sum_{i, j=1}^{M} k_{i j}\left\|u_{i} u_{j}\right\|_{p+1}^{p+1}=\sum_{i=1}^{M}\left\|\nabla u_{i}\right\|_{2}^{2}+\left\|u_{i}\right\|_{2}^{2}>0 .
$$

Therefore (P1) is equivalent to the existence of ground-states. To prove this, we did not use Schwarz symmetrization, since such an approach would only work if the coupling coefficients were positive. A careful application of the concentration-compactness principle turns out to be the right answer. Moreover, under fairly large conditions, we characterized the set of ground-states. More precisely, if one may group the components in such a way that two components attract each other if and only if they are in the same group, then only one of these groups is nontrivial, and it must have the same profile as the ground-state for the scalar equation.

Regarding stability, the scalar case was been treated in $[1,3,6]$, among others. For ground-states, stability is equivalent to the condition $p<2 / N$ (called the subcritical case). Note that, from the gauge and translation invariances, one should study the orbital stability of ground-states (that is, modulo rotations and translations). In [1], it is possible to find examples which show that one must really consider this kind of stability.

For the general case of bound-states, the problem is much more difficult. It can be seen that, assuming non-degeneracy, the orbital stability of a boundstates is directly related with the Morse index of the action at the bound-state (see [3]). If this index is 1 , then stability is again equivalent to the condition $p<2 / N$. If the index is greater than 1 , the problem remains open. Note that the assumption of non-degeneracy is not always true, even for ground-states: in [2], we proved that, for $M=2, k_{11}=k_{12}=k_{22}=1$ and $p=1$, there exist a continuum of ground-states which are not related by gauge invariance. This situation, though somewhat excepcional, shows that one cannot use a priori the results of [3]. 
In this work, we show the analogous stability results for ground-states of (M-NLS), assuming only (P1). This was done for $M=2$ and $k_{i j}>0$ in [5]. The framework will be very close to the scalar case as is [1], though some subtle changes will be done. Specifically, to prove stability (or instability), one proves that the set of ground-states is the set of minimizers of an adequate minimization problem. This is done in two steps:

1. Prove that the minimization problem has a solution, independently of the existence of ground-states;

2. Using the solution found in the previous step, show the equivalence between ground-states and minimizers.

Here, we change the argument. We shall prove directly that ground-states are minimizers and conclude the equivalence. This is more efficient, since the proof of existence of minimizers without using the ground-states and assuming only (P1) has to go through the concentration-compactness principle, which is not trivial at all (see [2]). Furthermore, we define three different classes of boundstates and prove stability results for these solutions. These are generalizations of the results obtained in [5].

\section{Definitions and main results}

Given any $U=\left(u_{1}, \ldots, u_{M}\right) \in\left(H^{1}\left(\mathbb{R}^{N}\right)\right)^{M}$, define the following functionals:

$$
\begin{gathered}
M(U):=\sum_{i=1}^{M}\left\|u_{i}\right\|_{2}^{2}, \quad T(U):=\sum_{i=1}^{M}\left\|\nabla u_{i}\right\|_{2}^{2}, \quad J(U):=\sum_{i, j=1}^{M} k_{i j}\left\|u_{i} u_{j}\right\|_{p+1}^{p+1}, \\
I(U):=M(U)+T(U), \quad E(U):=\frac{1}{2} T(U)-\frac{1}{2 p+2} J(U) \\
H(U):=T(U)-\frac{N p}{2 p+2} J(U) .
\end{gathered}
$$

Finally, define the action of $U$

$$
S(U)=\frac{1}{2} I(U)-\frac{1}{2 p+2} J(U) .
$$

Remark 1. The functional $M$ is called mass, $T$ is the kinetic energy and $J$ is the potential energy. Obviously, $E$ is the total energy (or just energy). Notice that (M-NLS) may be written in a Hamiltonian way:

$$
i U_{t}=E^{\prime}(U) \text {. }
$$

From this, one easily observes the conservation of the $L^{2}$-norm of each component (and therefore of the mass) and of the energy for (M-NLS): multiply (2.4) by $i U$ and $U_{t}$, respectively, take the real part and sum in $i$ for the latter case.

Remark 2. Consider, for $U \in\left(H^{1}\left(\mathbb{R}^{N}\right)\right)^{M}$ and $\lambda>0, \mathcal{P}(U, \lambda)(x)=\lambda^{\frac{N}{2}} U(\lambda x)$. By a change of variables, one sees that

$$
M(\mathcal{P}(U, \lambda))=M(U)
$$


Now, differentiating $S(\mathcal{P}(U, \lambda))$ with respect to $\lambda$,

$$
\frac{d}{d \lambda} S(\mathcal{P}(U, \lambda))=H(\mathcal{P}(U, \lambda))
$$

Remark 3. As in the scalar case, one may prove the Virial identity for (MNLS): given $V=\left(v_{1}, \ldots, v_{M}\right):[0, T) \rightarrow\left(H^{1}\left(\mathbb{R}^{N}\right)\right)^{M}$ solution of (M-NLS), one has

$$
\frac{d^{2}}{d t^{2}} \sum_{i=1}^{M}\left\|x v_{i}(t)\right\|_{2}^{2}=8 H(V(t)) .
$$

The quantity $\sum_{i=1}^{M}\left\|x v_{i}(t)\right\|_{2}^{2}$ is called the variance of $V(t)$. This identity will be essential when proving instability.

Definition 1. We say that $U \in\left(H^{1}\left(\mathbb{R}^{N}\right)\right)^{M}$ is a bound-state of (M-NLS) if it is a nonzero solution of (1.1). Furthermore, $U$ is a ground-state if $S(U) \leqslant S(W)$, for any bound-state $W$. The set of bound-states (resp. ground-states) will be noted by $A$ (resp. G).

Remark 4. If $U \in A$, then, multiplying (1.1) by $U$ and integrating over $\mathbb{R}^{N}$, $I(U)=J(U)$. Moreover, from Pohozaev's identity,

$$
H(U)=0 \text {. }
$$

This may also be readily seen from the Virial identity.

Definition 2. We note by $R$ the set of bound-states such that all nonzero components are equal to the same ground-state of (NLS), up to scalar multiplication and rotation.

Definition 3. Fix $X \subset\{1, \ldots, M\}$. An element $U \in A$ belongs to $G_{X}$ if the vector of its nonzero components is a ground-state for the (L-NLS) system for by the $i$-th components, with $i \in X$ and $L=|X|$.

Remark 5. It is known (see [1]) that, up to rotations and translations, there exists a unique ground-state for (NLS), which we note by $Q$. An element in $R$ must therefore be of the form

$$
U=\left(a_{i} e^{i \theta_{i}} Q(\cdot+y)\right),
$$

for some $a_{i} \geqslant 0, \theta_{i} \in \mathbb{R}$ and $y \in \mathbb{R}^{N}$.

Now we present some results of [2] that wil be used later.

Lemma 4. Assume (P1). Define

$$
\lambda_{G}:=\left(\inf _{J(U)=1} I(U)\right)^{\frac{p+1}{p}}>0 .
$$

Then the minimization problem

$$
I(U)=\min _{J(W)=\lambda_{G}} I(W), \quad J(U)=\lambda_{G}
$$

has a solution and $G$ is the set of its solutions. Moreover, any minimizing sequence strongly converges to an element in $G$. 
Lemma 5. Suppose (P1) and that there exists a partition $\left\{Y_{k}\right\}_{1 \leqslant k \leqslant K}$ of $\{1, \ldots, M\}$ such that, given $1 \leqslant i \neq j \leqslant M$,

$$
k_{i j} \geqslant 0 \quad \text { if and only if } \exists k: i, j \in Y_{k} .
$$

Then, if $U^{0}=\left(u_{1}^{0}, \ldots, u_{M}^{0}\right) \in G$, there exists $k \in\{1, \ldots, K\}$ such that $u_{i}^{0}=$ $0, \forall i \notin Y_{k}$ and $G \subset R$.

Remark 6. Without the hypothesis in the above lemma, there may exist situations where $R$ is empty.

Lemma 6. The optimal constant for the vector-valued Gagliardo-Nirenberg inequality

$$
J(W) \leqslant C M(W)^{p+1-\frac{N p}{2}} T(W)^{\frac{N p}{2}}, W \in\left(H^{1}\left(\mathbb{R}^{N}\right)\right)^{M}
$$

$i s$

$$
C_{M}=\frac{J(\mathcal{Q})}{M(\mathcal{Q})^{p+1-\frac{N p}{2}} T(\mathcal{Q})^{\frac{N p}{2}}}, \mathcal{Q} \in G .
$$

Moreover, one has equality if and only if

$$
\nu W(\zeta x) \in G,
$$

where

$$
\nu=\left(\frac{J(\mathcal{Q}) M(W)}{M(\mathcal{Q}) J(W)}\right)^{\frac{1}{2 p}}
$$

and

$$
\zeta=\left(\nu^{2}\left(\frac{M(W)}{M(\mathcal{Q})}\right)\right)^{\frac{1}{N}} .
$$

When searching for ground-states for (NLS), one may adopt two strategies: the first is the one presented in Lemma 4; the second is to minimize the energy, fixing the mass equal to some constant. Then, using a suitable scaling determined by the associated Lagrange multiplier, one obtains a ground-state. For a precise value of this constant, the multiplier is 1 and so minimizers are ground-states. Note that this only works if $p<2 / N$.

We can try to adopt a similar strategy for the (M-NLS) system, for $p<2 / N$. There are two ways of extending such a procedure:

- Minimize the energy, fixing the total mass equal to some constant. We show (Lemma 15) that this is equivalent to the minimization problem (2.11);

- Minimize the energy, fixing the mass of each component equal to some positive constant. More precisely, given $c>0$, consider the minimization problem

$$
E(U)=\min _{\left\{W:\left\|w_{i}\right\|_{2}^{2}=c\right\}} E(W), \quad\left\|u_{i}\right\|_{2}^{2}=c \quad \forall i .
$$

However, it is not necessary that one even obtains bound-states, since there will exist $M$ Lagrange multipliers which may be different, and so it is not possible to make a scaling to obtain a ground-state (notice that the minimizers will correspond to periodic solutions of the form $U=$ 
$\left(e^{i \omega_{i} t} u_{i}\right)_{1 \leqslant i \leqslant M}$. If $\omega_{i} \neq \omega_{j}$, the corresponding components will be out of phase).

Definition 7. We define $B^{c}$ to be the set of minimizers of (2.18) that belong to $A$. For $X \in\{1, \ldots, M\}$, we define $B_{X}^{c}$ to be the set of elements for which the vector of its nonzero components is in $B^{c}$ for the (L-NLS) system formed by the $i$-th components, with $i \in X$ and $L=|X|$.

Definition 8. Let $\mathcal{S} \subset\left(H^{1}\left(\mathbb{R}^{N}\right)\right)^{M}$ be invariant by the flow generated by (MNLS). We say that $\mathcal{S}$ is:

1. stable if, for each $\delta>0$, there exists an $\epsilon>0$ such that, for any $V_{0} \in$ $\left(H^{1}\left(\mathbb{R}^{N}\right)\right)^{M}$ with

$$
\inf _{W \in \mathcal{S}}\left\|V_{0}-W\right\|_{H^{1}\left(\mathbb{R}^{N}\right)^{M}}<\epsilon,
$$

the solution $V$ of (M-NLS) with initial data $V_{0}$ satisfies

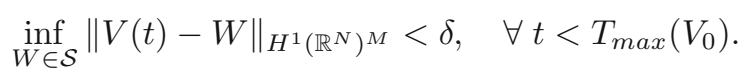

2. weakly unstable if there exist $\epsilon>0$ and a sequence $V_{n}^{0}$ such that

$$
\inf _{U \in \mathcal{S}}\left\|V_{n}^{0}-U\right\|_{H^{1}\left(\mathbb{R}^{N}\right)^{M}} \rightarrow 0, \quad n \rightarrow \infty
$$

and, letting $V_{n}$ be the solution of (M-NLS) with initial data $V_{n}^{0}$,

$$
\sup _{t \in\left[0, T_{\max }\left(V_{n}^{0}\right)\right)} \inf _{U \in \mathcal{S}}\left\|V_{n}(t)-U\right\|_{H^{1}\left(\mathbb{R}^{N}\right)^{M}}>\epsilon .
$$

3. unstable if, for any $U \in \mathcal{S}$, there exists a sequence $U_{n} \rightarrow U$ such that $T_{\max }\left(U_{n}\right)<\infty$, for any $n \in \mathbb{N}$.

Next, we present the main results of this paper:

Theorem 9. Assume $(\mathrm{P} 1)$ and $p<2 / N$. For any $X \subset\{1, \ldots, M\}$, let $G_{1} \subset$ $G_{X}$ be such that $\operatorname{dist}\left(G_{1}, G_{X} \backslash G_{1}\right)>\delta$, for some $\delta>0$. Then $G_{1}$ is stable.

Remark 7. In many cases, the set $G$ is discrete modulo translations and rotations. Then any connected component of $G$ is stable. Since these components are obtained by translations and rotations of a given element, one obtains orbital stability of ground-states.

Proposition 10. Suppose (P1), $p<2 / N, k_{i j}>0, i \neq j$ and that there exists $\beta>0$ such that

$$
\sum_{j=1}^{M} k_{i j}=\beta, \quad \forall i .
$$

Then, for $c=\left\|\beta^{\frac{1}{2 p}} Q\right\|_{2}^{2}$,

$$
B^{c}=\left\{\left(e^{i \theta_{i}} \beta^{\frac{1}{2 p}} Q(\cdot+y)\right)_{1 \leqslant i \leqslant M}: \theta_{i} \in \mathbb{R}, y \in \mathbb{R}^{N}\right\} \subset R
$$

and, given $X \subset\{1, \ldots, M\}, B_{X}^{c}$ is stable.

Remark 8. The above results show the existence of stable bound-states that are not ground-states. 
Theorem 11. Assume (P1) and $p>2 / N$. Then $G$ and $R$ are unstable.

Remark 9. Under the assumptions of lemma 5, it is sufficient to prove that $R$ is unstable, and this follows from the instability of the ground-states for (NLS).

Theorem 12. Assume $(\mathrm{P} 1)$ and $p>2 / N$. If $U \in A$ is a local minimum of $S$ over the set

$$
\mathcal{H}:=\{W: H(W)=0\},
$$

then the set $\left\{e^{i \theta} U(\cdot+y): \theta \in \mathbb{R}, y \in \mathbb{R}^{N}\right\}$, is weakly unstable.

Remark 10. Set $p>2 / N$. Assuming that $U \in A$ is a non-degenerate critical point of the action (modulo rotations), the Morse index of $S$ at $U, m(U)$, is greater or equal to 1: a negative direction is given by the path $\lambda \mapsto S(\mathcal{P}(U, \lambda))$. Note that this direction does not belong to the tangent space of $\mathcal{H}$ at $U$. Therefore, the condition in the above theorem is equivalent $m(U)=1$. The problem for $m(U) \geqslant 2$ is much more difficult, and it is still unanswered for the scalar equation.

Theorem 13. Assume $(\mathrm{P} 1)$ and $p=2 / N$. Then $A$ is unstable.

\section{Stability in the subcritical case}

Throughout this section, we shall assume $p<2 / N$.

Lemma 14. There exists $\mu>0$ such that

$$
M(\mathcal{Q})=\mu, \quad \forall \mathcal{Q} \in G .
$$

Proof. This follows easily from the identities $I(\mathcal{Q})=J(\mathcal{Q})$ and Pohozaev's identity.

Lemma 15. Assume (P1). Then $G$ is the set of of solutions of the minimization problem

$$
E(U)=\min _{M(W)=\mu} E(W), \quad M(U)=\mu .
$$

Moreover, if $\left\{W_{n}\right\}$ is a minimizing sequence, then $J\left(W_{n}\right) \rightarrow J(\mathcal{Q})$, with $\mathcal{Q} \in$ $G$.

Proof. Let $W$ be such that $M(W)=\mu$. Consider the function (see remark 2)

$$
\lambda \mapsto f(\lambda)=E(\mathcal{P}(W, \lambda)), \quad \lambda>0
$$

Since $p<2 / N, f$ has a unique minimum $\lambda_{0}$. Let $Z=f\left(\lambda_{0}\right)$. Then $f^{\prime}\left(\lambda_{0}\right)=0$, which implies that $H(Z)=0$, i.e.,

$$
T(Z)=\frac{N p}{2 p+2} J(Z) .
$$

Therefore,

$$
E(Z)=\frac{N p-2}{2 N p} T(Z) .
$$


Using the vector-valued Gagliardo-Nirenberg inequality,

$$
\frac{2 p+2}{N p} T(Z)=J(Z) \leqslant C_{M} M(Z)^{\frac{2-(N-2) p}{2}} T(Z)^{\frac{N p}{2}},
$$

and so, from $M(Z)=\mu$,

$$
\frac{2 p+2}{N p} T(Z)^{\frac{2-N p}{2}} \leqslant C_{M} \nu^{\frac{2-(N-2) p}{2}} .
$$

Let $\mathcal{Q} \in G$. By lemma 6 , we obtain $T(Z) \leqslant T(\mathcal{Q})$. Therefore

$$
E(W) \geqslant E(Z)=\frac{N p-2}{2 N p} T(Z) \geqslant \frac{N p-2}{2 N p} T(\mathcal{Q})=E(\mathcal{Q})
$$

and so $\mathcal{Q}$ is a solution of (3.1). If $W$ is also a solution of (3.1), then one must have equality in (3.5). Again by lemma 6 ,

$$
\nu W(\zeta x) \in G
$$

with $\nu, \zeta$ given by $(2.16),(2.17)$. Since $M(W)=M(\mathcal{Q})$ and $J(W)=J(\mathcal{Q})$, $\nu=\zeta=1$. Therefore $W \in G$.

If $\left\{W_{n}\right\}_{n \in \mathbb{N}}$ is a minimizing sequence, define $\left\{Z_{n}\right\}_{n \in \mathbb{N}}$ as above. Then $\left\{Z_{n}\right\}$ is also a minimizing sequence and

$$
\left\|W_{n}-Z_{n}\right\|_{\left(H^{1}\left(\mathbb{R}^{N}\right)\right)^{M}} \rightarrow 0, \quad n \rightarrow \infty .
$$

Hence

$$
\begin{aligned}
\frac{N p-2}{2(2 p+2)} J(\mathcal{Q}) & =E(\mathcal{Q})=\lim E\left(Z_{n}\right)=\lim \frac{N p-2}{2(2 p+2)} J\left(Z_{n}\right) \\
& =\lim \frac{N p-2}{2(2 p+2)} J\left(W_{n}\right),
\end{aligned}
$$

as we wanted.

Proof of Theorem 9: We start with the stability for $X=\{1, \ldots, M\}$ (that is, for $G)$. By contradiction suppose that there exists a sequence $\left\{V_{n}^{0}\right\}_{n \in \mathbb{N}} \subset$ $\left(H^{1}\left(\mathbb{R}^{N}\right)\right)^{M}$ such that, for some $\mathcal{Q}_{0} \in G_{1}$,

$$
\left\|V_{n}^{0}-\mathcal{Q}_{0}\right\|_{\left(H^{1}\left(\mathbb{R}^{N}\right)\right)^{M}} \rightarrow 0, \quad n \rightarrow \infty
$$

and, letting $V_{n}$ be the solution of (M-NLS) with initial data $V_{n}^{0}$, there exist $\left\{t_{n}\right\}_{n \in \mathbb{N}}$ and $\epsilon>0$ such that

$$
\inf _{\mathcal{Q} \in G_{1}}\left\|V_{n}\left(t_{n}\right)-\mathcal{Q}\right\|_{\left(H^{1}\left(\mathbb{R}^{N}\right)\right)^{M}}=\epsilon .
$$

By continuity and conservation of mass and energy,

$$
E\left(V_{n}\left(t_{n}\right)\right)=E\left(V_{n}^{0}\right) \rightarrow E\left(\mathcal{Q}_{0}\right), M\left(V_{n}\left(t_{n}\right)\right)=M\left(V_{n}^{0}\right) \rightarrow M\left(\mathcal{Q}_{0}\right)=\mu .
$$

Therefore, the sequence

$$
W_{n}=\left(\frac{\mu}{M\left(V_{n}^{0}\right)}\right)^{\frac{1}{2}} V_{n}\left(t_{n}\right)
$$

is a minimizing sequence of (3.1). By lemma $15, J\left(W_{n}\right) \rightarrow J\left(\mathcal{Q}_{0}\right)$. From lemma $4, W_{n} \rightarrow \mathcal{Q}_{1}$, with $\mathcal{Q}_{1} \in G$, which implies that $V_{n}\left(t_{n}\right) \rightarrow \mathcal{Q}_{1}$. Taking $\epsilon<\delta$, one obtains $d\left(\mathcal{Q}_{1}, G_{1}\right)<\delta$, which means that $\mathcal{Q}_{1} \in G_{1}$, which is absurd. 
In the general case, given $X \subset\{1, \ldots, M\}$, one may proceed exactly as above: for $i \notin X$, since the mass of each component is conserved, the $i$ th components must converge to 0 in $L^{2}$ and, by interpolation, to 0 in $L^{2 p+2}$. This means that the remaining components are a minimizing sequence of (3.1), for the (L-NLS) system formed by the components in $X$, and therefore must converge to a ground-state of such a system.

Proof of proposition 10: As in the previous proof, we start with $X=$ $\{1, \ldots, M\}$. The general case $X \subset\{1, \ldots, M\}$ is treated as in the previous proof.

Define, for $u \in H^{1}\left(\mathbb{R}^{N}\right)$,

$$
E_{1}(u)=\frac{1}{2}\|\nabla u\|_{2}^{2}-\frac{\beta}{2 p+2}\|u\|_{2 p+2}^{2 p+2} .
$$

By lemma 15 for $M=1$, the set of solutions of the minimization problem

$$
E_{1}(u)=\min _{\|w\|_{2}^{2}=\| \beta^{\frac{1}{2 p}}} Q\left\|_{2}^{2} E_{1}(w), \quad\right\| u\left\|_{2}^{2}=\right\| \beta^{\frac{1}{2 p}} Q \|_{2}^{2}
$$

is $\left\{e^{i \theta} Q(\cdot+y): \theta \in \mathbb{R}, y \in \mathbb{R}^{N}\right\}$. Let $U=\left(u_{1}, \ldots, u_{M}\right) \in\left(H^{1}\left(\mathbb{R}^{N}\right)\right)^{M}$ be such that $\left\|u_{i}\right\|_{2}^{2}=\left\|\beta^{\frac{1}{2 p}} Q\right\|_{2}^{2}$. Then

$$
\sum_{i=1}^{M} E_{1}\left(u_{i}\right) \geqslant \sum_{i=1}^{M} E_{1}(Q) .
$$

Let $\mathcal{Q}$ be the vector formed by $M$ copies of $Q$. Now, from Young's inequality, we have

$$
E(U) \geqslant \sum_{i=1}^{M} E_{1}\left(u_{i}\right) \geqslant \sum_{i=1}^{M} E_{1}(Q)=E(\mathcal{Q}) .
$$

Therefore $\mathcal{Q}$ is a solution of (2.18). If $U$ is also a solution, one must have equality in the above relation, which implies that

$$
u_{i}=e^{i \theta_{i}} Q\left(\cdot+y_{i}\right), \quad \theta_{i} \in \mathbb{R}, \quad y_{i} \in \mathbb{R}^{N} .
$$

If there exist $i_{0}, j_{0}$ such that $y_{i_{0}} \neq y_{j_{0}}$, one easily sees that there exists $D \subset \mathbb{R}^{N}$ of positive measure such that, for all $x \in D, Q\left(x+y_{i_{0}}\right) \neq Q\left(x+y_{j_{0}}\right)$ and so, using Young's inequality,

$$
Q\left(x+y_{i_{0}}\right)^{p+1} Q\left(x+y_{j_{0}}\right)^{p+1}<\frac{1}{2} Q\left(x+y_{i_{0}}\right)^{2 p+2}+\frac{1}{2} Q\left(x+y_{j_{0}}\right)^{2 p+2}, \quad x \in D .
$$

On the other hand, we have in general

$$
\begin{aligned}
& Q\left(x+y_{i}\right)^{p+1} Q\left(x+y_{j}\right)^{p+1} \leqslant \frac{1}{2} Q\left(x+y_{i}\right)^{2 p+2}+\frac{1}{2} Q\left(x+y_{j}\right)^{2 p+2}, \\
& x \in \mathbb{R}^{N}, \quad 1 \leqslant i, j \leqslant M .
\end{aligned}
$$


Consequently,

$$
\begin{aligned}
& \int\left(a_{i} Q\left(\cdot+y_{i}\right)\right)^{p+1}\left(a_{j} Q\left(\cdot+y_{j}\right)\right)^{p+1} \\
& \leqslant a_{i}^{p+1} a_{j}^{p+1}\left(\frac{1}{2} \int Q\left(\cdot+y_{i}\right)^{2 p+2}+\frac{1}{2} \int Q\left(\cdot+y_{j}\right)^{2 p+2}\right) \\
& =a_{i}^{p+1} a_{j}^{p+1} \int Q^{2 p+2}=\int\left(a_{i} Q\right)^{p+1}\left(a_{j} Q\right)^{p+1},
\end{aligned}
$$

with strict inequality if $i=i_{0}$ and $j=j_{0}$ and so

$$
E(U)=\frac{1}{2} T(U)-\frac{1}{2 p+2} J(U)>\frac{1}{2} T(\mathcal{Q})-\frac{1}{2 p+2} J(\mathcal{Q})=E(\mathcal{Q}),
$$

which is absurd. Hence

$$
B^{c}=\left\{\left(e^{i \theta_{i}} \beta^{\frac{1}{2 p}} Q(\cdot+y)\right)_{1 \leqslant i \leqslant M}: \theta_{i} \in \mathbb{R}, y \in \mathbb{R}^{N}\right\} .
$$

Now we prove the stability property. By contradiction, suppose that there exists a sequence $\left\{V_{n}^{0}\right\}_{n \in \mathbb{N}} \subset\left(H^{1}\left(\mathbb{R}^{N}\right)\right)^{M}$ such that, for some $\mathcal{Q}_{0} \in G$,

$$
\left\|V_{n}^{0}-\mathcal{Q}_{0}\right\|_{\left(H^{1}\left(\mathbb{R}^{N}\right)\right)^{M}} \rightarrow 0, \quad n \rightarrow \infty
$$

and, letting $V_{n}$ be the solution of (M-NLS) with initial data $V_{n}^{0}$, there exist $\left\{t_{n}\right\}_{n \in \mathbb{N}}$ and $\epsilon>0$ such that

$$
\inf _{\mathcal{Q} \in B^{c}}\left\|V_{n}\left(t_{n}\right)-\mathcal{Q}\right\|_{\left(H^{1}\left(\mathbb{R}^{N}\right)\right)^{M}}>\epsilon .
$$

By continuity and from the conservation of the $L^{2}$ norm of each component and of the energy,

$$
E\left(V_{n}\left(t_{n}\right)\right)=E\left(V_{n}^{0}\right) \rightarrow E\left(\mathcal{Q}_{0}\right),\left\|\left(V_{n}\left(t_{n}\right)\right)_{i}\right\|_{2}^{2}=\left\|\left(V_{n}^{0}\right)_{i}\right\|_{2}^{2} \rightarrow\left\|\beta^{\frac{1}{2 p}} Q\right\|_{2}^{2} .
$$

Therefore, the sequence $W_{n}=\left(w_{n}^{1}, \ldots, w_{n}^{M}\right)$ defined by

$$
w_{n}^{i}=\left(\frac{\left\|\beta^{\frac{1}{2 p}} Q\right\|_{2}^{2}}{\left\|\left(V_{n}^{0}\right)_{i}\right\|_{2}^{2}}\right)^{\frac{1}{2}} V_{n}\left(t_{n}\right), \quad i=1, \ldots, M
$$

is a minimizing sequence of (2.18). Now notice that this implies that

$$
E_{1}\left(w_{n}^{i}\right) \rightarrow E_{1}(Q), \quad i=1, \ldots, M
$$

From the stability results for (NLS) (see [1], chapter 8), this implies that, for some $\theta_{i} \in \mathbb{R}$ and $y_{i} \in \mathbb{R}^{N}, w_{n}^{i} \rightarrow e^{i \theta_{i}} Q\left(\cdot+y_{i}\right)$. Applying a reasoning as before, we see that $y_{i}=y$, for all $i$. Therefore $W_{n} \rightarrow \mathcal{Q}_{1}$, with $\mathcal{Q}_{1} \in B^{c}$ and so $V_{n} \rightarrow \mathcal{Q}_{1}$, which is absurd, by (3.24).

\section{Instability in the supercritical case}

In this section, we study the case $p>2 / N$. We define, for $W \neq 0, \lambda^{*}(W)$ to be the maximum of the function $g(\lambda)=S(\mathcal{P}(W, \lambda))$. 
Lemma 16. Assume (P1). Then $G$ is the set of of solutions of the minimization problem

$$
S(U)=\min _{H(W)=0} S(W), \quad H(U)=0 .
$$

Proof. Let $W$ be such that $H(W)=0$ and consider the function (see remark 2)

$$
\lambda \mapsto g(\lambda)=S(\mathcal{P}(W, \lambda)), \quad \lambda>0
$$

Since $p>2 / N$, this function has a unique maximum and, by (2.6), it must be $\lambda=1$. Therefore

$$
S(\mathcal{P}(\lambda, W)) \leqslant S(W), \quad \forall \lambda>0
$$

On the other hand, there exists $\lambda_{0}>0$ such that $J\left(\mathcal{P}\left(\lambda_{0}, U\right)\right)=\lambda_{G}$. Hence, for $\mathcal{Q} \in G$,

$$
S(\mathcal{Q}) \leqslant S\left(\mathcal{P}\left(\lambda_{0}, W\right)\right) \leqslant S(W), \quad \forall W: H(W)=0 .
$$

Therefore $G$ is a subset of the set of solutions of (4.1) and the latter is nonempty.

Now consider $U$ solution of (4.1). Define, for $\sigma>0, U_{\sigma}(x)=\sigma^{\frac{1}{p}} U(\sigma x)$. By a change of variables,

$$
H\left(U_{\sigma}\right)=\sigma^{2-N+\frac{2}{p}} H(U)=0 .
$$

Since $U$ is a minimizer, one must have

$$
\left.\frac{d}{d \sigma} S\left(U_{\sigma}\right)\right|_{\sigma=1}=0, \quad \text { i.e. }\left\langle S^{\prime}(U), U\right\rangle_{H^{-1} \times H^{1}}=0
$$

On the other hand, there exists $\eta$ such that $S^{\prime}(U)=\eta H^{\prime}(U)$. Applying to $U$ and using $H(U)=0$,

$$
0=\left\langle S^{\prime}(U), U\right\rangle_{H^{-1} \times H^{1}}=\eta\left\langle H^{\prime}(U), U\right\rangle_{H^{-1} \times H^{1}}=-2 p \eta T(U) .
$$

Therefore $\eta=0$ and so $U \in A$. Given $\mathcal{Q} \in G, H(\mathcal{Q})=0$, and so $S(U) \leqslant S(\mathcal{Q})$, which means that $U \in G$.

Lemma 17. Let $\mathcal{Q} \in G$ and $W \in\left(H^{1}\left(\mathbb{R}^{N}\right)\right)^{M}$ such that $H(W)<0$. Then

$$
H(W) \leqslant S(W)-S(\mathcal{Q}) .
$$

Proof. Once again, consider the function

$$
\lambda \mapsto g(\lambda)=S(\mathcal{P}(W, \lambda)), \quad \lambda>0 .
$$

This function has a maximum $\lambda_{0}<1$ ( since $H(W)<0$ ) and is concave in $\left(\lambda_{0}, 1\right)$. Therefore, by remark 2.6 ,

$S(W) \geqslant S\left(\mathcal{P}\left(W, \lambda_{0}\right)\right)+\left(1-\lambda_{0}\right) H(W) \geqslant S\left(\mathcal{P}\left(W, \lambda_{0}\right)\right)+H(W) \geqslant S(\mathcal{Q})+H(W)$,

since $H\left(\mathcal{P}\left(W, \lambda_{0}\right)\right)=0$ and $\mathcal{Q}$ is a solution of $(4.1)$.

Remark 11. More generally, given any $U \in\left(H^{1}\left(\mathbb{R}^{N}\right)\right)^{M}$, one may prove as above that, if $W$ is such that $H(W)<0$ and $S\left(\mathcal{P}\left(W, \lambda^{*}(W)\right)\right) \geqslant S(U)$,

$$
H(W) \leqslant S(W)-S(U) .
$$


Proof of Theorem 11: Firstly, we prove that $G$ is unstable. Consider a groundstate $\mathcal{Q}$. Then, for any $\lambda>1, \mathcal{Q}_{\lambda}:=\mathcal{P}(\mathcal{Q}, \lambda)$ satisfies

$$
H\left(\mathcal{Q}_{\lambda}\right)<0 .
$$

Let $V_{\lambda}$ be the solution of (M-NLS) with initial data $\mathcal{Q}_{\lambda}$. For $t$ small, $H\left(V_{\lambda}(t)\right)<0$. From the conservation of mass and energy,

$$
S\left(V_{\lambda}(t)\right)=S\left(\mathcal{Q}_{\lambda}\right) .
$$

By the previous lemma, for any $t$ such that $H\left(V_{\lambda}(t)\right)<0$, one has

$$
H\left(V_{\lambda}(t)\right) \leqslant S\left(V_{\lambda}(t)\right)-S(\mathcal{Q}) \leqslant S\left(\mathcal{Q}_{\lambda}\right)-S(\mathcal{Q})=-\delta<0 .
$$

Therefore, by continuity, one must have $H\left(V_{\lambda}(t)\right) \leqslant-\delta, \forall t<T_{\max }\left(\mathcal{Q}_{\lambda}\right)$. Now, using (2.7),

$$
\frac{d^{2}}{d t^{2}} \sum_{i=1}^{M}\left\|x\left(v_{\lambda}\right)_{i}(t)\right\|_{2}^{2}=8 H(V(t))<-8 \delta .
$$

Since the variance is positive, one must have $T_{\max }\left(\mathcal{Q}_{\lambda}\right)<\infty$ and so $G$ is unstable.

If $U=\left(u_{1}, \ldots, u_{M}\right) \in R$, then there exist $a_{i} \geqslant 0, \theta_{i} \in \mathbb{R}$ and $y \in \mathbb{R}^{N}$ such that $u_{i}=a_{i} e^{i \theta_{i}} Q(\cdot+y)$. Since $Q(\cdot+y)$ is a ground-state for (1-NLS), there exists a sequence $\left\{v_{n}^{0}\right\}_{n \in \mathbb{N}}$ such that $v_{n}^{0} \rightarrow Q(\cdot+y)$ in $H^{1}\left(\mathbb{R}^{N}\right)$ and $T_{\max }\left(v_{n}^{0}\right)<\infty, \forall n$. Let $v_{n}$ be the solution of (1-NLS) with initial data $v_{n}^{0}$. Then one can observe that $V_{n}=\left(a_{i} e^{i \theta_{i}} v_{n}\right)_{1 \leqslant i \leqslant M}$ is a solution of (M-NLS), with initial data $V_{n}^{0}=\left(a_{i} e^{i \theta_{i}} v_{n}^{0}\right)_{1 \leqslant i \leqslant M}$. Since $V_{n}^{0} \rightarrow U$ in $\left(H^{1}\left(\mathbb{R}^{N}\right)\right)^{M}$, one concludes that $R$ is unstable.

Proof of Theorem 12: Let $U \in A$ be a local minimum of $S$ restricted to $\mathcal{H}$. Let $B_{\delta}(U)$ be a ball with center at $U$ and radius $\delta$ fixed such that

$$
S(U) \leqslant S(W), \quad \forall W \in B_{\delta}(U) \cap \mathcal{H} .
$$

For $\epsilon>0$ small, one has

$$
\mathcal{P}\left(W, \lambda^{*}(W)\right) \in B_{\delta}(U), \quad \forall W \in B_{\epsilon}(U) .
$$

From remark 11, if $W \in B_{\epsilon}(U)$ is such that $H(W)<0$,

$$
H(W) \leqslant S(W)-S(U) .
$$

Notice that, from the invariance of $S$ and $H$ regarding rotations and translations, the same remains valid for

$$
W \in \Sigma:=\left\{e^{i \theta} Z(\cdot+y): \theta \in \mathbb{R}, y \in \mathbb{R}^{N}, \quad Z \in B_{\epsilon}(U)\right\} .
$$

Consider $U_{\lambda}=\mathcal{P}(U, \lambda), \lambda>1$. Then $H\left(U_{\lambda}\right)<0$. Let $V_{\lambda}$ be the solution of (M-NLS) with initial data $U_{\lambda}$. If $V_{\lambda}(t) \in \Sigma, \forall t<T_{\max }\left(U_{\lambda}\right)$, then, arguing as in the previous proof,

$$
H\left(V_{\lambda}(t)\right) \leqslant S\left(U_{\lambda}\right)-S(U)=-\delta<0, \quad \forall t<T_{\max }\left(U_{\lambda}\right) .
$$

Then (4.14) is valid, which leads to $T_{\max }\left(U_{\lambda}\right)<\infty$. Since $\Sigma$ is bounded, we arrive at a contradiction. 


\section{Instability in the critical case}

Proof of Theorem 13: First, notice that $2 E(W)=H(W)$, for any $W \in$ $\left(H^{1}\left(\mathbb{R}^{N}\right)\right)^{M}$. Let $U \in A$. Then $2 E(U)=H(U)=0$. For any $\lambda>1, H(\lambda U)<0$. Since $2 E=H$, the conservation of energy implies that, setting $V_{\lambda}$ to be the solution of (M-NLS) with initial data $\lambda U, H\left(V_{\lambda}(t)\right)=H(\lambda U), t<T_{\max }(\lambda U)$. One now concludes as in the supercritical case, using the Virial identity.

\section{Further comments}

If one considers periodic solutions of (M-NLS) of the form $V=\left(e^{i \omega_{1} t} u_{1}, \ldots\right.$, $\left.e^{i \omega_{M} t} u_{M}\right)$, with $U=\left(u_{1}, \ldots, u_{M}\right) \in\left(H^{1}\left(\mathbb{R}^{N}\right)\right)^{M}$, we arrive to the system

$$
\Delta u_{i}-\omega_{i} u_{i}+\sum_{j=1}^{M} k_{i j}\left|u_{j}\right|^{p+1}\left|u_{i}\right|^{p-1} u_{i}=0 \quad i=1, \ldots, M .
$$

This is a generalization of the notion of bound-state that was presented in this work, where components may be out of phase. This implies that a simple characterization of solutions (cf. lemma 5) of 6.1 based on $Q$ are impossible. On the other hand, results regarding stability of bound-state that do not use any characterization are imediately extendible to this broader notion of boundstate, since the only influence of the parameters $\omega_{i}$ (which is in the definition of $I$ and $M$ ) does not alter the properties of these functionals.

\section{Acknowledgements}

This work was supported by Fundação para a Ciência e Tecnologia, Portugal, through the Grant SFRH/BD/96399/2013. We thank Mário Figueira for his helpful comments and suggestions.

\section{References}

[1] Cazenave, T.: Semilinear Schrödinger equations. Courant Institute of Mathematical Sciences New York (2003)

[2] Correia, S.: Characterization of ground-states for a system of M coupled semilinear Schrödinger equations. arXiv:1410.7993 (pre-print)

[3] Grillakis, M., Shatah, J., Strauss, W.: Stability theory of solitary waves in the presence of symmetry I. J. Func. Anal 74, 160-197 (1987)

[4] Lions, P.-L.: The concentration-compactness principle in the Calculus of Variations. The locally compact case, part I. Ann. Inst. Henri Poincaré 2, 109 145 (1984)

[5] Maia, L.A.; Montefusco, E.; Pellacci, B.: Orbital stability property for coupled nonlinear Schrödinger equations. arXiv:0809.3320 (pre-print) 
[6] Shatah, J., Strauss, W.: Instability of bound states. Comm. Math. Phys 100, 173190 (1985)

Simão Correia

CMAF-UL and FCUL

Campo Grande

1749-016 Lisboa

Portugal

e-mail: sfcorreia@fc.ul.pt

Received: 13 May 2015.

Accepted: 3 March 2016. 